\title{
Low-scale neutrino seesaw mechanism and scalar dark matter
}

\author{
M. Fabbrichesi ${ }^{1, a}$, S. T. Petcov ${ }^{1,2,3}$ \\ ${ }^{1}$ INFN, Sezione di Trieste, Trieste, Italy \\ ${ }^{2}$ SISSA, via Bonomea 265, 34136 Trieste, Italy \\ ${ }^{3}$ Kavli IPMU, University of Tokyo, Tokyo, Japan
}

Received: 13 December 2013 / Accepted: 5 February 2014 / Published online: 20 February 2014

(c) The Author(s) 2014. This article is published with open access at Springerlink.com

\begin{abstract}
We discuss how two birds- the little hierarchy problem of low-scale type-I seesaw models and the search for a viable dark matter candidate—are (proverbially) killed by one stone: a new inert scalar state.
\end{abstract}

\section{Motivations}

Together with the presence of dark matter (DM), neutrino oscillations - and the small neutrino mass entailed - are the only physics beyond the standard model (SM) experimentally confirmed.

The most attractive model to account for the smallness of the neutrino masses is the seesaw mechanism [1-4]. This mechanism requires right-handed $(\mathrm{RH})$ neutrinos whose masses can be taken at the GUT scale or, in low-scale scenarios, at lower energies if the Yukawa couplings are taken proportionally smaller, for instance, of the order of those of the charged leptons.

The inclusion of these new states within the SM induces a finite renormalization that tends to pull the Higgs boson mass (or, equivalently, the electroweak (EW) scale) toward the higher scale. This is not a problem if the new states are themselves at the EW scale and the renormalization is itself of the order of the Higgs boson mass. If instead the new states are at a larger scale, they give rise to a hierarchy problem in which in order to keep the Higgs boson mass to its experimental value we have to cancel the renormalization to the higher scale to a degree that becomes increasingly fine-tuned as the new states are taken at higher mass scales.

Such a cancelation can be achieved by a redefinition of the Higgs boson bare mass but it is more appealing and practical to use the hierarchy problem in a heuristic manner to help us in the definition of whatever model of physics we assume to exist beyond the SM [5].

\footnotetext{
a e-mail: marco@sissa.it
}

The problem of the large contribution to the Higgs mass $m_{\mathrm{H}}$ coming from the new states is best understood in terms of the renormalization group equation (RGE)

$\mu \frac{\mathrm{d} m_{\mathrm{H}}}{\mathrm{d} \mu}=-m_{\mathrm{H}} \gamma_{m_{\mathrm{H}}}^{(1)}$,

where the anomalous dimension is given, at one-loop order, by

$\gamma_{m_{\mathrm{H}}}^{(1)}=-\frac{1}{16 \pi^{2}}\left(6 \lambda+3 \lambda_{\mathrm{t}}^{2}-\frac{9}{4} g_{2}^{2}-\frac{3}{2} g_{1}^{2}\right)$,

in dimensional regularization and where we only wrote the dominant contributions from the Higgs boson potential $(\lambda)$, the top quark $\left(\lambda_{t}\right)$ and the gauge couplings $\left(g_{1}\right.$ and $\left.g_{2}\right)$. If new states are present at a higher scale, they must be introduced as a threshold effect in order to match the low- and highscale effective theories. That these threshold corrections are larger and larger as the new states are taken to be heavier and heavier is the hierarchy problem in the framework of an effective theory.

According to the proposed approach, the new stateswhen they are taken together-must enter in such a way that their overall effect on the Higgs boson mass renormalization is no larger than the EW scale, thus making the bare mass renormalization natural. In other words, using the RGE terminology, the threshold corrections must be small and if the new states are very heavy they must enter in such a way as to cancel their respective contributions.

The fine-tuning, if present, is all among the new states rather than between them and the SM contributions. In this approach it is possible to keep the physics at the two different scales separated so as to make possible an effective theory treatment.

This point of view is suggested by the idea that this is what would happen if the hierarchy problem had been ameliorated by the presence of a (possibly broken) symmetry. Of course, in our case there is no such a symmetry but the identification of the states necessary for controlling the size of the 
renormalization might eventually lead to the identification of one.

We discuss the case in which the masses of the RH neutrinos are in the range between one and a few hundreds of TeVs. The renormalization effects are in this case dominated by the one-loop order and we have what has been called the little hierarchy problem. To avoid this problem, new states in addition to the RH neutrinos must be included. As a matter of fact, the addition of one new state is enough. We study its properties and to what extent is a viable candidates for DM. This way, two birds (the little hierarchy problem and DM) are killed by one stone (the new state) and two a priori unrelated physical problems are nicely brought together. The fine-tuning required by the cancelation is around $10 \%$.

To avoid confusion, let us stress that the hierarchy problem is often discussed in terms of the quadratic divergence arising in the mass term of the Higgs boson in a momentum dependent regularization (or, equivalently, in a pole in $d=2$ dimensions in dimensional regularization). The presence of these divergences makes the Higgs boson mass extremely sensitive to the UV physics and some cancelation must take place either in a natural manner by assuming a symmetry (usually, supersymmetry) or by fine-tuning by imposing the Veltman condition [6] (see, also, [7-9]) — namely that the new sector couples to the SM Higgs boson just so as to make the quadratic divergences to the SM Higgs boson mass vanish (see [10-15] for various applications of this idea). This is not the hierarchy problem we discuss in this Letter.

The point of view we follow is that all quadratic divergencies are a scheme-dependent artifact which obfuscates the problem by making impossible to separate degrees of freedom living at different energy scales. One can even argue that in the Higgs boson mass case, they arise because of the explicit breaking of scale invariance in momentum dependent regularizations (in a way similar to the quadratic divergence arising in QED when we take a momentum dependent regularization which violates gauge symmetry), and they should be eliminated by an appropriate counterterm [16-19] or by not using that particular regularization scheme.

The point is that, even without these divergent terms, there are large finite renormalization effects which only depends on integrating out the heavy modes in the low-energy effective theory - the SM in our case. We identify the little hierarchy problem with the presence of these finite terms. These corrections are similar to those arising in a supersymmetric theory with soft mass terms where the quadratic divergencies are canceled while, after integrating out the heavy states, there are finite terms whose contribution shifts the values of the Higgs boson mass [20,21]. This work is about these terms in the case of the seesaw mechanism.

\section{Low-scale seesaw and the little hierarchy problem}

We consider a type I seesaw model in which three $\mathrm{RH}$ neutrinos $N_{a R}$ are added to the SM as $S U(2)_{L}$ singlets. The lagrangian of the model is given by the kinetic and Yukawa terms of the SM with the addition of the neutrino Yukawa terms:

$\mathcal{L}=-y_{a \ell}^{v} \bar{N}_{a R} \tilde{H}^{\dagger} L_{\ell}-\frac{1}{2} \bar{N}_{a L}^{c} M_{\mathrm{N} a b} N_{b R}+H . c .$,

where $L_{\ell}$ represents the SM left-handed $S U(2)$ doublet $\left(v_{\ell}, \ell\right)_{L}$ and $\ell=e, \mu, \tau$. In Eq. (3), the Yukawa term gives rise to the neutrino Dirac mass matrix, $M_{D}=y v_{W}$, after the Higgs field $H=\left(v_{W}+h\right)$ takes its vacuum expectation value $v_{W}=174 \mathrm{GeV}$. The heavy RH neutrinos $N_{a R}$ have a Majorana mass term.

We compute the one-loop finite correction to the Higgs boson mass using dimensional regularization with renormalization scale $\mu$. The SM particle contributions are negligible. To compute the one-loop renormalization arising from the heavy Majorana neutrinos, we rotate the Yukawa couplings $y_{a l}^{v}$ into the basis in which the heavy RH neutrino mass matrix $M_{\mathrm{N}}$ is diagonal. In this basis the matrix of neutrino Yukawa couplings takes the following form in the type I seesaw model of interest [22,23]:

$\hat{y}_{j \ell}^{v}=M_{\mathrm{N}_{j}}(R V)_{j \ell}^{\mathrm{T}} / v_{W}$

where $V$ is a unitary matrix which diagonalises the RH neutrino Majorana mass matrix, $M_{\mathrm{N}}=V \hat{M} V^{\mathrm{T}}$ with $\hat{M}=$ $\operatorname{diag}\left(M_{1}, M_{2}, M_{3}\right), M_{j}$ being the mass of the heavy neutrino mass-eigenstate $N_{j}$, and $R^{\mathrm{T}} \cong M_{\mathrm{N}}^{-1} M_{D}\left(\left|M_{D}\right| \ll\left|M_{\mathrm{N}}\right|\right)$. As can be shown (see, e.g., [22]), the quantity $(R V)_{\ell j}$ represents the weak charged current and neutral current coupling of the heavy Majorana neutrino $N_{j}$ to the charged lepton $l$ and the $W^{ \pm}$-bosons, and to the LH flavor neutrino $\nu_{l}$ and the $Z^{0}$-boson. The matrix $\eta=-0.5(R V)(R V)^{\dagger}$ describes, in the seesaw model considered, the deviations from unitarity of the Pontecorvo, Maki, Nakagawa, Sakata (PMNS) neutrino mixing matrix $U_{\mathrm{PMNS}}$ : $U_{\mathrm{PMNS}}=(1+\eta) U$, where $U$ is a unitary matrix which diagonalises the Majorana mass matrix of the LH flavor neutrinos, $m_{v}$, generated by the seesaw mechanism.

In the type I seesaw scenario, the elements of the matrix $(R V)$ are bounded by their relation to the elements of the neutrino mass matrix $m_{v}$ [22], which all have to be smaller than approximately $1 \mathrm{eV}$ :

$\left|\sum_{k}(R V)_{\ell^{\prime} k}^{*} M_{k}(R V)_{k \ell}^{\dagger}\right|=\left|\left(m_{v}\right)_{\ell^{\prime} \ell}\right| \lesssim 1 \mathrm{eV}$.

In the traditional seesaw model the Yukawa couplings are taken typically to be of order one and the masses $M_{\mathrm{N}_{j}}$ are very large, close to the GUT scale. The couplings $\left|(R V)_{\ell k}\right|$ in this case have to be very small to satisfy Eq. (5). In low-scale 
seesaw models, the heavy Majorana neutrino masses lie at the $\mathrm{TeV}$ scale and the couplings $\left|(R V)_{\ell k}\right|$ are proportionally larger. In this scenario, $\left|(R V)_{\ell k}\right|$ can even be larger if there is partial or complete cancelation between the terms in the sum in the r.h.s. of Eq. (5). This possibility can be realized, e.g., in models [24] with two heavy Majorana neutrinos, $N_{1}$ and $N_{2}$, which have relatively close masses, $M_{2}=M_{1}(1+z)$, $z \ll 1$, thus forming a pseudo-Dirac state [25-27], and whose couplings $(R V)_{\ell 1}$ and $(R V)_{\ell 2}$ are related: $(R V)_{\ell 2} \sqrt{M_{2}}=$ $\pm i(R V)_{\ell 1} \sqrt{M_{1}}, l=e, \mu, \tau$. The indicated conditions can take place, for instance, in theories with an approximately conserved lepton charge (see, e.g., [28]).

In the scenario with two heavy Majorana neutrinos outlined above, the flavor structure of the couplings $(R V)_{\ell j}$, $j=1,2$, is completely determined by the requirement of reproducing the neutrino oscillation data and the scheme is characterized by four parameters [24]: $M_{1}, z$, the largest eigenvalue $y$ of the matrix of neutrino Yukawa couplings (see further) and a CP violation phase. The neutrino oscillation data, the EW precision measurements and the existing limits on the rates of lepton flavor violating (LFV) processes involving the charged leptons (as the $\mu \rightarrow e+\gamma, \mu \rightarrow 3 e$ decays, etc.), imply the following upper bounds on the couplings $\left|(R V)_{\ell 1}\right| \cong\left|(R V)_{\ell 2}\right|$ (see, e.g., $[29,30]$ and references quoted therein):

$\left|(R V)_{e 1}\right|^{2},\left|(R V)_{\mu 1}\right|^{2},\left|(R V)_{\tau 1}\right|^{2} \lesssim 10^{-3}$

where we have quoted a somewhat simplified constraint on the three couplings. The actual upper bounds depend on the flavor index $l$ of the couplings, but the variation with $l$ is not significant and for the purposes of our investigation it can be neglected. We will use the generic bounds given in Eq. (6) in our analysis.

In what follows we will neglect for simplicity the splitting between the two heavy Majorana neutrino masses $z$, i.e., we will set $z=0$ and will use $M_{1}=M_{2} \equiv M_{\mathrm{N}}$. The corrections due to $z \neq 0$ are insignificant in the problem of interest. For $z=0$, the largest eigenvalue $y$ of the matrix of neutrino Yukawa couplings is given by [24]

$$
y^{2} v_{W}^{2}=2 M_{\mathrm{N}}^{2}\left[\left|(R V)_{e 1}\right|^{2}+\left|(R V)_{\mu 1}\right|^{2}+\left|(R V)_{\tau 1}\right|^{2}\right]
$$

Taking into account the one-loop contribution and assuming RH neutrino degeneracy, the Higgs boson mass receives a shift given by

$\delta \mu_{\mathrm{H}}^{2}(\mu)=\frac{4 y^{2}}{(4 \pi)^{2}} M_{\mathrm{N}}^{2}\left(1-\log \frac{M_{\mathrm{N}}^{2}}{\mu^{2}}\right)$,

being $\mu$ the matching scale that in this case we can identify with $M_{\mathrm{N}}$.

The addition of the RH neutrinos would shift the Higgs boson mass to the new scale unless we balance this new contribution to prevent large one-loop renormalizations. The identification of what states (their masses and couplings to the Higgs boson) must be present for such a balancing act to occur provides the heuristic power of the little hierarchy problem.

While many possible new states can be added to prevent large corrections to the Higgs boson mass, the simplest choice consists in including just an inert scalar state [31-38], that is, a scalar particle only interacting with the Higgs boson (and gravity) - and therefore transforming as the singlet representation of the EW gauge group $S U(2) \times U(1)$ (and similarly not charged under the color group) — which acquires no vacuum expectation value. Such a choice minimizes unwanted effects on EW radiative corrections and other physics well described by the SM.

If in addition we impose a $Z_{2}$ symmetry under which the inert scalar is odd and all the SM fields are even, the new state will couple to the SM Higgs doublet only through quartic interactions in the scalar potential. By construction, as we only look for solutions with vanishing vacuum expectation value, the symmetry $Z_{2}$ remains unbroken and after $\mathrm{EW}$ symmetry breaking the singlet state can, as we shall discuss, potentially be a viable cold DM candidate.

The scalar potential of the model is given by

$$
\begin{aligned}
V(H, S)= & \mu_{\mathrm{H}}^{2}\left(H^{\dagger} H\right)+\mu_{\mathrm{S}}^{2} S^{2} \\
& +\lambda_{1}\left(H^{\dagger} H\right)^{2}+\lambda_{2} S^{4}+\lambda_{3}\left(H^{\dagger} H\right) S S .
\end{aligned}
$$

Linear and trilinear terms for $S$ are absent due to the $Z_{2}$ symmetry mentioned above.

Taking into account the one-loop contribution induced by the scalar state $S$, the overall shift to $\mu_{\mathrm{H}}^{2}$, taking $M_{\mathrm{S}}<M_{\mathrm{N}}$ and $\mu=M_{\mathrm{S}}$ to minimize the logarithmic contributions to the matching, becomes

$\delta \mu_{\mathrm{H}}^{2}\left(M_{\mathrm{S}}\right)=\frac{1}{(4 \pi)^{2}}\left[\lambda_{3} M_{\mathrm{S}}^{2}-4 y^{2} M_{\mathrm{N}}^{2}\left(1-\log \frac{M_{\mathrm{N}}^{2}}{M_{\mathrm{S}}^{2}}\right)\right]$,

where we have taken $y$ given in Eq. (7) for the Yukawa couplings.

We want the threshold correction in Eq. (10) to be of the order of the Higgs boson mass itself. For simplicity, we can just impose $\delta \mu_{\mathrm{H}}^{2}=0$ and obtain

$\lambda_{3}=\frac{4 y^{2} M_{\mathrm{N}}^{2}}{M_{\mathrm{S}}^{2}}\left(1-\log \frac{M_{\mathrm{N}}^{2}}{M_{\mathrm{S}}^{2}}\right)$.

Equation (11) is economical but we must bear in mind that it represents just the special case in which the one-loop renormalization exactly vanishes. More solutions can easily be found for $\delta \mu_{\mathrm{H}}^{2} \simeq m_{H}^{2}$ but do not change in a significant manner the numerical results.

Because of the extra factor $1 /(4 \pi)^{2}$, two-loop corrections become important only if the masses are above $10 \mathrm{TeV}$. To be 
safe, we take the matching scale $\mu=M_{\mathrm{S}}$ smaller than $7 \mathrm{TeV}$. For these values, the fine-tuning of requiring $\delta \mu_{\mathrm{H}}^{2} \simeq m_{H}^{2}$ is about $10 \%$.

\section{Enter dark matter}

It has been shown [33-38] that a single inert singlet that couples with the Higgs boson with a small coupling is a realistic cold weakly interacting DM candidate (WIMP) with a mass below $v_{W}$. In our case, the singlet may account for the correct relic density in the opposite regime where its mass is much larger than $v_{W}$ and its coupling with the Higgs boson relatively large.

In this case, the scattering amplitude is dominated by the point-like $S S \rightarrow h h$ vertex which gives a contribution to the thermally averaged total cross section equal to

$\langle\sigma v\rangle \simeq \frac{1}{4 \pi} \frac{\lambda_{3}^{2}}{M_{\mathrm{S}}^{2}} \sqrt{1-\frac{m_{H}^{2}}{m_{\mathrm{S}}^{2}}}$,

where we keep only the s-wave contribution.

To estimate the viability of $S$ as DM candidate, we must compute its relative relic abundance [39-42],

$\Omega_{\mathrm{S}}=\frac{M_{\mathrm{S}} n_{\mathrm{S}}\left(t_{0}\right)}{\rho_{c}}$

where $\rho_{c}=1.05 h^{2} 10^{-5} \mathrm{GeV} / \mathrm{cm}^{3}$ and the density $n_{\mathrm{S}}\left(t_{0}\right)$ is given by

$n_{\mathrm{S}}\left(t_{0}\right)=\sqrt{\frac{45}{\pi g_{*}}} \frac{s_{0}}{M_{\mathrm{pl}} T_{\mathrm{f}}\langle\sigma v\rangle}$,

where $M_{\mathrm{pl}}$ is the Planck mass, $T_{\mathrm{f}}$ is the freeze-out temperature, which for our and similar candidates is approximately given by

$m_{\mathrm{S}} / T_{\mathrm{f}} \simeq \log \frac{M_{\mathrm{S}} M_{\mathrm{pl}}\langle\sigma v\rangle}{240 \sqrt{g_{*}}} \sim 26$,

and $s_{0}=2.8 \times 10^{3} \mathrm{~cm}^{-3}$ is the entropy density. The constant $g_{*}=106.75+1$ counts the number of SM degrees of freedom in thermal equilibrium plus the additional degrees of freedom related to the singlet.

We therefore obtain

$\Omega_{\mathrm{S}} h^{2} \simeq 8.41 \times 10^{-11} \frac{M_{\mathrm{S}}}{T_{\mathrm{f}}} \sqrt{\frac{45}{\pi g_{*}}} \frac{\mathrm{GeV}^{-2}}{\langle\sigma v\rangle}$,

which is sufficiently accurate for our purposes.

Current data fit within the standard cosmological model give a relic abundance with $\Omega_{\mathrm{DM}} h^{2}=0.1187 \pm 0.0017$ [43]. By combining the central value above with Eqs. (12) and (16), we can write the coupling $\lambda_{3}$ as function of $M_{\mathrm{S}}$ thus obtaining $\left|\lambda_{3}\right| \simeq 0.15 \frac{M_{\mathrm{S}}}{\mathrm{TeV}}$.

This solution gives a DM candidate which can account for $100 \%$ of the relic density and with a cross section of a few $\mathrm{pb}$ which makes it weakly interacting, a WIMP.

The presence of the scalar singlet DM improves the EW vacuum stability with respect to the SM [44]. This is particularly interesting in connection with the presence of the RH neutrinos which, in general, have the opposite effect of reducing the stability region of the Higgs boson potential [45].

Insertion of Eq. (17) in Eq. (11) gives a relationship between the RH neutrino mass and that of the new scalar if the latter is to be considered a viable candidate for DM:

$0.15 M_{\mathrm{S}}^{3}=8 \alpha \frac{M_{\mathrm{N}}^{4}}{v_{W}^{2}}\left(1-\log \frac{M_{\mathrm{N}}^{2}}{M_{\mathrm{S}}^{2}}\right)$

where $\alpha=\left|(R V)_{e 1}\right|^{2}+\left|(R V)_{\mu 1}\right|^{2}+\left|(R V)_{\tau 1}\right|^{2}$ represents the sum of the squares of the couplings of the $\mathrm{RH}$ and $\mathrm{LH}$ neutrinos. In Eq. (18), all masses must be taken in TeVs.

Possible solutions are shown in Fig. 1. We can see that we can have solutions with $M_{\mathrm{S}} \simeq M_{\mathrm{N}}$ as long as we take the couplings between LH and RH neutrinos, and therefore $\alpha$, to be as large as possible within the experimental constraints in Eq. (6). This case corresponds to taking the largest Yukawa coupling just at its experimental bound. This is the most interesting range because the RH neutrino masses are still in a range accessible to the experiments (e.g., $\mu \rightarrow$ $e+\gamma, \mu \rightarrow 3 e$ decays, $\mu^{-}-e^{-}$conversion in nuclei, neutrinoless double $\beta$-decay).

On the other hand, if these couplings are taken at their natural values (and no cancelation is assumed in their sum) we can have only solutions where $M_{\mathrm{S}}$ is much smaller than $M_{\mathrm{N}}$ because now the Yukawa couplings are much smaller than 1 . In this case, $\lambda_{3}$ becomes negative and one has to check that $\lambda_{3} \geq-2 \sqrt{\lambda_{1} \lambda_{2}}$ for the stability of the potential. This scenario seems less interesting than the previous one because the RH neutrino masses are several hundreds of $\mathrm{TeVs}$ and therefore outside the range of any foreseeable experiment.

It is interesting to notice that there exists another regime in which the inert singlet is a viable DM candidate. When $\lambda_{3}$ is much smaller than 1 , thermal equilibrium for the scalar states is never attained and their abundance is so low that they never annihilate among themselves. The usual result does not apply. In this case, the DM candidate is what has been called a feebly interacting massive particle (FIMP). If we do not use Eq. (17) and take $\lambda_{3} \simeq 10^{-11}[46,47]$, we can have a DM candidate in which $M_{\mathrm{S}} \simeq M_{\mathrm{N}}$ (see Fig. 2). For these solutions, $\alpha \simeq 10^{-12}$ and the Yukawa couplings become of the order of those of the charged leptons.

Let us briefly comment on the possibility of detecting the inert scalar $S$ in the case in which is a WIMP. 

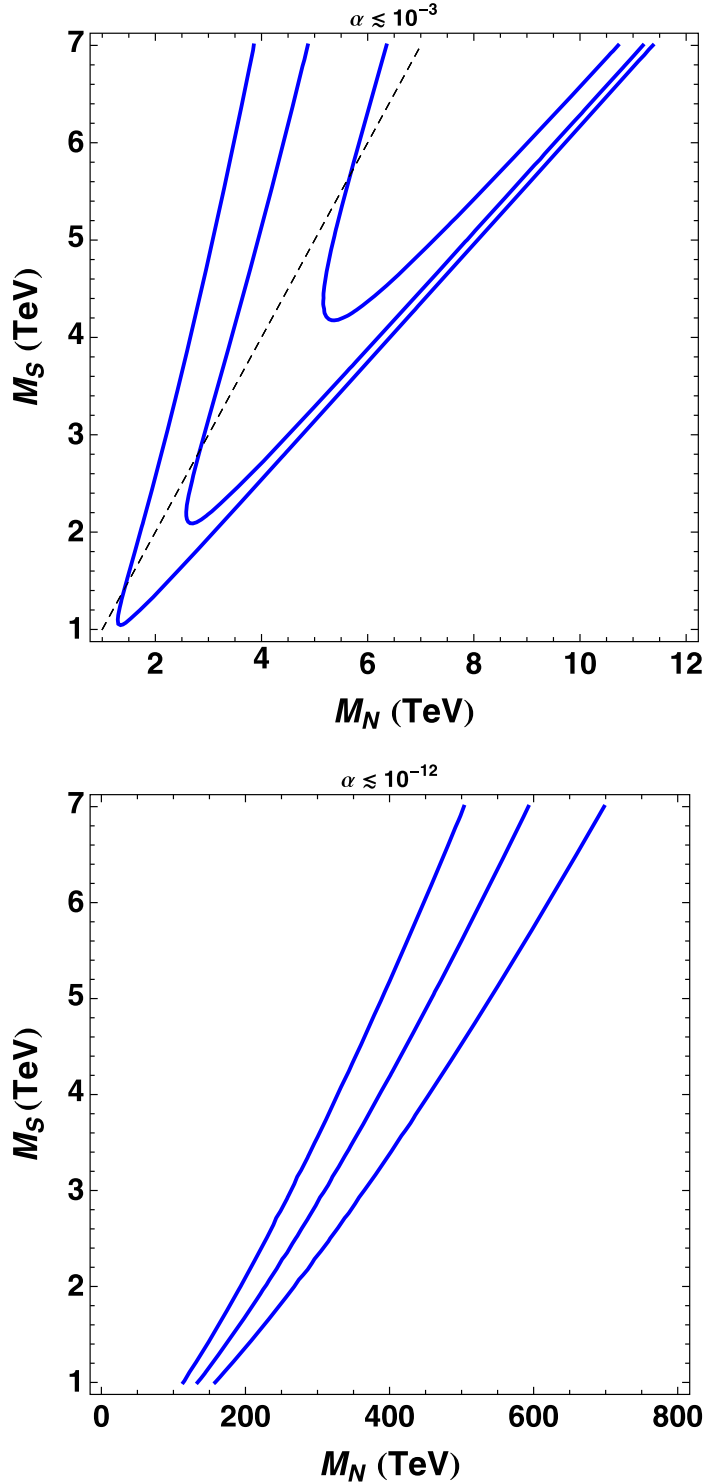

Fig. 1 Contour plot for the WIMP solution: values of $M_{\mathrm{S}}$ and $M_{\mathrm{N}}$ that are solutions of Eq. (18) for two limiting sets of values of the parameter $\alpha$. On top (in descending order) $\alpha=\{1,2,4\} \times 10^{-4}$, below $\alpha=\{1,2,4\} \times 10^{-13}$. Solutions with $m_{\mathrm{S}}<m_{\mathrm{N}}$ are below the dashed line in the figure on top

The multi $\mathrm{TeV}$ range of its mass makes its detection at the LHC very difficult if not impossible.

Similarly, we do not expect this DM candidate to be constrained by current experiments in space (PAMELA, FERMI, AMS2 etc.). Its mass is larger than $1 \mathrm{TeV}$ with a weakly interacting cross section that is too small to produce an enhancement in the $100 \mathrm{GeV}$ range in annihilation processes with positrons or $\gamma$-rays in the final states [48,49]. Moreover, because it only interacts with the Higgs boson, it produces positron and photons mainly through hadronic states $\left(\pi^{+}\right.$and $\pi^{0}$, respectively) with a corresponding broad energy spec-

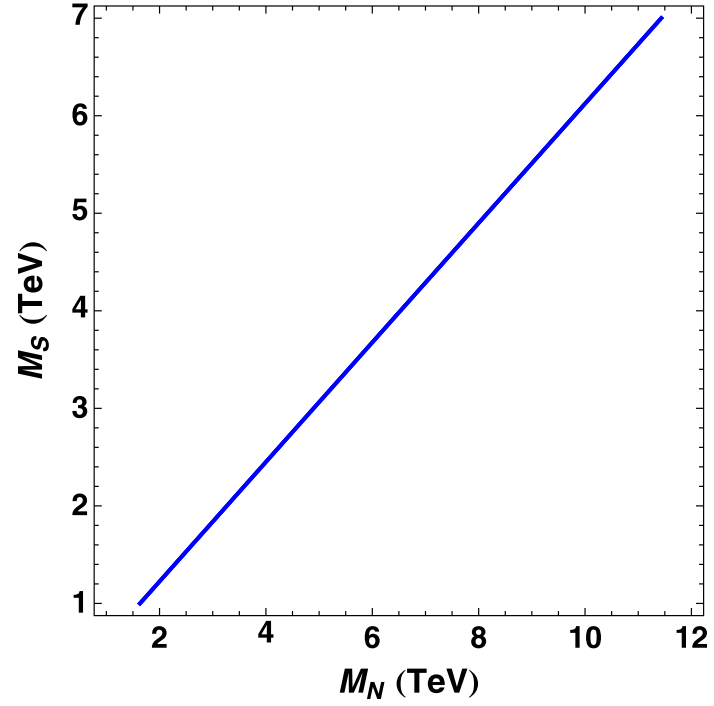

Fig. 2 Contour plot for the FIMP solution: values of $M_{\mathrm{S}}$ and $M_{\mathrm{N}}$ that are solutions of Eq. (11) in the case of $\lambda_{3} \simeq 10^{-11}$ and $\alpha \simeq 10^{-12}$

trum which would be difficult to disentangle from that of other sources.

Nuclear scattering experiments are a more promising place where to look for a possible experimental signature. The quartic term proportional to $\lambda_{3}$ in Eq. (9) gives rise, after EW symmetry breaking, to the three-field interaction $S S h$, which yields the effective singlet-nucleon vertex $f_{\mathrm{N}} \lambda_{3} m_{\mathrm{N}} / m_{H}^{2} S S \bar{\psi}_{\mathrm{N}} \psi_{\mathrm{N}}$, where $m_{\mathrm{N}}$ is the nucleon mass and the factor $f_{\mathrm{N}}$ contains many uncertainties due to the computation of the nuclear matrix elements, and it can vary from 0.3 to $0.6[50,51]$. The (non-relativistic) cross section for the process is given by $[52,53]$

$\sigma_{\mathrm{N}}=f_{\mathrm{N}}^{2} m_{\mathrm{N}}^{2} \frac{\lambda_{3}^{2}}{4 \pi}\left(\frac{m_{\mathrm{r}}}{m_{\mathrm{S}} m_{H}^{2}}\right)^{2}$,

where $m_{\mathrm{r}}$ is the reduced mass for the system which is, to a vary good approximation in our case, equal to the nucleon mass $m_{\mathrm{N}}$. Substituting the values we have found for our model and for $m_{\mathrm{S}}$ of a few $\mathrm{TeV}$, we obtain, depending on the choice of parameters and within the given uncertainties, a cross section $\sigma_{\mathrm{N}}$ of order $10^{-45} \mathrm{~cm}^{2}$, a value within reach of the next generation of experiments $[54,55]$.

Acknowledgments This research was supported in part by the World Premier International Research Center Initiative (WPI Initiative), MEXT, Japan, by the European Union FP7-ITN INVISIBLES (Marie Curie Action, PITAN-GA-2011-289442) and by the INFN program on "Astroparticle Physics" (S.T.P.).

Open Access This article is distributed under the terms of the Creative Commons Attribution License which permits any use, distribution, and reproduction in any medium, provided the original author(s) and the source are credited.

Funded by $\mathrm{SCOAP}^{3}$ / License Version CC BY 4.0. 


\section{References}

1. P. Minkowski, Phys. Lett. B 67, 421 (1977)

2. M. Gell-Mann, P. Ramond, R. Slansky, Conf. Proc. C790927, 315321 (1979). Print-80-0576. arXiv: 1306.4669

3. T. Yanagida, Conf. Proc. C7902131, 95-99 (1979). KEK-79-1095, Workshop on Baryon Number of the Universe and Unified Theories, ed. by Sawada and Sagumoto

4. R.N. Mohapatra, G. Senjanovic, Phys. Rev. Lett. 44, 91 (1980)

5. F. Bazzocchi, M. Fabbrichesi, Phys. Rev. D 87, 036001 (2013). arXiv: 1212.5065

6. M.J.G. Veltman, Acta Phys. Polon. B 12, 437 (1981)

7. P. Osland, T.T. Wu, Phys. Lett. B 291, 315 (1992)

8. E. Ma, Phys. Rev. D 47, 2143 (1993). arXiv:hep-ph/9209221

9. G. Ossola, A. Sirlin, Eur. Phys. J. C 31, 165 (2003). arXiv:hep-ph/ 0305050

10. A. Kundu, S. Raychaudhuri, Phys. Rev. D 53, 4042 (1996). arXiv: hep-ph/9410291

11. N.G. Deshpande, R.J. Johnson, E. Ma, Phys. Lett. B 130, 61 (1983)

12. F. Bazzocchi, M. Fabbrichesi, P. Ullio, Phys. Rev. D 75, 056004 (2007). arXiv:hep-ph/0612280

13. B. Grzadkowski, J. Wudka, Phys. Rev. Lett. 103, 091802 (2009). arXiv:0902.0628

14. A. Drozd, B. Grzadkowski, J. Wudka, JHEP 1204, 006 (2012). arXiv: 1112.2582

15. F. Bazzocchi, M. Fabbrichesi, Eur. Phys. J. C 73, 2303 (2013). arXiv:1207.095

16. W.A. Bardeen, FERMILAB-CONF-95-391-T

17. R. Foot, A. Kobakhidze, K.L. McDonald, R.R. Volkas, Phys. Rev. D 77, 035006 (2008). arXiv:0709.2750 [hep-ph]

18. K.A. Meissner, H. Nicolai, Phys. Lett. B 660, 260 (2008). arXiv: 0710.2840

19. M. Shaposhnikov, D. Zenhausern, Phys. Lett. B 671, 162 (2009). arXiv:0809.3406

20. See, e.g., J.A. Casas, J.R. Espinosa, M. Quiros, A. Riotto, Nucl. Phys. B 436, 3 (1995) [Erratum-ibid. B 439, 466 (1995)]. arXiv: hep-ph/9407389, and references therein

21. J.A. Casas, J.R. Espinosa, I. Hidalgo, JHEP 0411, 057 (2004). arXiv:hep-ph/0410298

22. A. Ibarra, E. Molinaro, S.T. Petcov, JHEP 1009, 108 (2010). arXiv: 1007.2378

23. C.G. Cely, A. Ibarra, E. Molinaro, S.T. Petcov, Phys. Lett. B 718, 957 (2013). arXiv:1208.3654

24. A. Ibarra, E. Molinaro, S.T. Petcov, Phys. Rev. D 84, 013005 (2011). arXiv:1103.6217

25. L. Wolfenstein, Nucl. Phys. B 186, 147 (1981)

26. S.T. Petcov, Phys. Lett. B 110, 245 (1982)

27. C.N. Leung, S.T. Petcov, Phys. Lett. B 125, 461 (1983)

28. M.B. Gavela, T. Hambye, D. Hernandez, P. Hernandez, JHEP 0909 , 038 (2009). arXiv:0906.1461

29. E. Akhmedov, A. Kartavtsev, M. Lindner, L. Michaels, J. Smirnov, JHEP 1305, 081 (2013). arXiv: 1302.1872
30. D.N. Dinh, A. Ibarra, E. Molinaro, S.T. Petcov, JHEP 1208, 125 (2012). arXiv:1205.4671

31. R. Barbieri, L.J. Hall, V.S. Rychkov, Phys. Rev. D 74, 015007 (2006). arXiv:hep-ph/0603188

32. V. Barger, P. Langacker, M. McCaskey, M.J. Ramsey-Musolf, G. Shaughnessy, Phys. Rev. D 77, 035005 (2008). arXiv:0706.4311 [hep-ph]

33. V. Silveira, A. Zee, Phys. Lett. B 161, 136 (1985)

34. J. McDonald, Phys. Rev. D 50, 3637 (1994). arXiv:hep-ph/ 0702143

35. C.P. Burgess, M. Pospelov, T. ter Veldhuis, Nucl. Phys. B 619, 709 (2001). arXiv:hep-ph/0011335

36. R. Dick, R.B. Mann, K.E. Wunderle, Nucl. Phys. B 805, 207 (2008). arXiv:0803.1444

37. C.E. Yaguna, JCAP 0903, 003 (2009). arXiv:0810.4267

38. K. Cheung, Y.-L.S. Tsai, P.-Y. Tseng, T.-C. Yuan, A. Zee, JCAP 1210, 042 (2012). arXiv:1207.4930 [hep-ph]

39. M. Srednicki, R. Watkins, K.A. Olive, Nucl. Phys. B 310, 693 (1988)

40. P. Gondolo, G. Gelmini, Nucl. Phys. B 360, 145 (1991)

41. M. Cirelli, N. Fornengo, A. Strumia, Nucl. Phys. B 753, 178 (2006) arXiv:hep-ph/0512090

42. D.S. Gorbunov, V.A. Rubakov, Introduction to the Theory of the Early Universe (World Scientific, Singapore, 2011)

43. P.A.R. Ade et al. [Planck Collaboration], arXiv:1303.5076 [astroph.CO]

44. M. Gonderinger, Y. Li, H. Patel, M.J. Ramsey-Musolf, JHEP 1001, 053 (2010). arXiv:0910.3167 [hep-ph]

45. J. Elias-Miro, J.R. Espinosa, G.F. Giudice, G. Isidori, A. Riotto, A. Strumia, Phys. Lett. B 709, 2 (2012). arXiv:1112.3022 [hep-ph]

46. C.E. Yaguna, JHEP 1108, 060 (2011). arXiv: 1105.1654

47. C.E. Yaguna, JCAP 1202, 006 (2012). arXiv:1111.6831

48. See, for example, M. Pohl, D. Eichler, arXiv:0912.1203 [astroph.HE]

49. L. Bergstrom, T. Bringmann, I. Cholis, D. Hooper, C. Weniger, Phys. Rev. Lett. 111, 171101 (2013). arXiv:1306.3983 [astroph.HE]

50. A. Bottino et al., Astropart. Phys. 13, 215 (2000). arXiv:hep-ph/ 9909228

51. A. Bottino et al., Astropart. Phys. 18, 205 (2002). arXiv:hep-ph/ 0111229

52. R. Barbieri, M. Frigeni, G.F. Giudice, Nucl. Phys. B 313, 725 (1989)

53. J.R. Ellis et al., Phys. Rev. D 71, 095007 (2005). arXiv:hep-ph/ 0502001

54. M. Schumann [XENON100 collaboration], J. Phys. Conf. Ser. 309, $012011(2011)$

55. P.L. Brink et al., [CDMS-II Collaboration], eConf C 041213, 2529 (2004). arXiv:astro-ph/0503583 and http://dmtools.brown.edu/ 